\title{
A PESQUISA-AÇÃO NA RECONSTRUÇÃO DO CONHECIMENTO DE ENFERMEIROS SOBRE PROCESSO DE ENFERMAGEM NA ÁREA HOSPITALAR
}

\author{
Rayanne Valentim Ribeiro', Flavia Nantes Fausto', Patrícia Trindade Benites², Arminda \\ Rezende de Pádua Del Corona ${ }^{2}$, e Vilma Ribeiro da Silva ${ }^{2}$ \\ ${ }^{1}$ Hospital Universitário Maria Aparecida Pedrossian, Brasil. ray_anne_14@hotmail.com; flavianantesfausto@gmail.com \\ ²Universidade Federal de Mato Grosso do Sul, Brasil. pattben@hotmail.com; delcorona.arp@gmail.com; \\ vrsilvams@yahoo.com.br
}

\begin{abstract}
Resumo. Introdução: O Processo de Enfermagem instrumentaliza a ação dos enfermeiros em direção ao cuidado individualizado e resolutivo ao cliente. Amparados por regulação própria, muitos profissionais buscam construir conhecimento e estruturar os serviços, de forma a promover a aplicação do Processo de Enfermagem em suas realidades, mas ainda encontram dificuldades para executá-lo. Objetivo: Conhecer o desenvolvimento das etapas de implantação do Processo de Enfermagem em um hospital. Método: Estudo qualitativo desenvolvido pelo método pesquisaação, com dados coletados aplicando-se instrumento semiestruturado a 87 enfermeiros assistenciais. Resultados: A Análise Temática permitiu identificar as seguintes categorias: Conhecimento do enfermeiro sobre o conceito de Sistematização da Assistência de Enfermagem; Etapas do Processo de Enfermagem; Indicação das fases do Processo de Enfermagem aplicadas diariamente; Habilidade na aplicação da classificação de Diagnóstico de Enfermagem; Fragilidades na aplicação do Processo de Enfermagem. Conclusões: Os enfermeiros indicaram os aspectos que fragilizaram a aplicação do Processo de Enfermagem quando apontaram sobrecarga de trabalho, deficiência no dimensionamento de pessoal e falta de sistema de informação que comportasse o registro do Processo de Enfermagem como fatores que prejudicaram seu desempenho.
\end{abstract}

Palavras-chave: Pesquisa-Ação; Processo de Enfermagem; Cuidados de Enfermagem. ACTION RESEARCH IN THE RECONSTRUCTION OF NURSES' KNOWLEDGE OF THE
NURSING PROCESS IN THE HOSPITAL AREA

\begin{abstract}
Background: The Nursing Process instrumentalizes nurses' action towards individualized and resolutive care to the client. With regulatory support, however, many professionals try to build knowledge and structure services, in order to promote the application of the Nursing Process in their realities, but they still find it difficult to execute. Objective: To know the development of the stages of implementation of the Nursing Process in a hospital. Method: Qualitative study developed by the action-research method, with data collected using a semi-structured instrument applied to 87 nursing assistants. Results: Thematic Analysis identified the following categories: Knowledge of nurses about the concept of Systematization of Nursing Assistance; Steps of the Nursing Process; Indication of the phases of the Nursing Process applied daily; Ability to apply the Nursing Diagnosis classification; Weaknesses in the application of the Nursing Process. Conclusions: The nurses indicated the aspects that weakened the application of the Nursing Process when they pointed out work overload, deficiency in the dimensioning of personnel and lack of an information system that would include the registration of the Nursing Process as factors that hindered their performance.
\end{abstract}

Keywords: Action Research; Nursing Process; Nursing Care.

\section{INTRODUÇÃO}

Com Florence Nightingale, a enfermagem iniciou sua caminhada rumo à adoção de uma prática baseada em conhecimentos científicos, abandonando gradativamente a postura de 
atividade caritativa, intuitiva e empírica (Friedlander, 1981), e ganhando, assim, na visão de muitos pesquisadores, o crédito da primeira teórica da enfermagem moderna.

Por um longo tempo, a enfermagem se desenvolveu tendo como referência o modelo nightingaleano. Enquanto isso, estudiosos de enfermagem realizaram trabalhos filosóficos e teóricos de enfermagem, permitindo evolução em vários estágios da teoria de enfermagem (McEwen \& Wills, 2016).

A partir das concepções e da validação das teorias, a enfermagem que, até então, era constituída por conhecimento clínico, estético, ético, somático e intuitivo, passa a ter seu corpus epistemológico formado (Caper, 1978). Adiante, Barret (2002) define a ciência de enfermagem como o "conhecimento substantivo, específico à disciplina, que enfoca o processo humano-universo-saúde articulado nas estruturas e teorias de enfermagem" ( $p$. 57).

Nesse movimento, a preocupação em estabelecer um método que instrumentalizasse a ação dos enfermeiros em direção ao cuidado individualizado ao cliente vem sendo percebida pela enfermagem há décadas. Desde 1929, nos Estados Unidos, e 1934, no Brasil, os estudos de caso foram introduzidos nas discussões de ensino e práticas. Estes estudos eram compostos, basicamente, pela história da doença, pela evolução da moléstia, pelo tratamento médico e pelos cuidados de enfermagem (Andrade \& Vieira, 2005).

As primeiras tentativas de sistematizar o cuidado de enfermagem, por meio da introdução do Processo de Enfermagem (PE) nos serviços de saúde e no ensino de graduação da enfermagem no Brasil, ocorreram no final da década de 1970 e início da década de 1980, com Wanda de Aguiar Horta, e possibilitaram ao enfermeiro a adoção de um método de trabalho sistematizado e baseado no conhecimento científico (Marques, Brito, Fernandes, \& Vieira, 2008). A partir de então, buscar construir um corpo de conhecimentos próprio e uma prática independente e autônoma parece representar as mais elevadas aspirações da enfermagem (Cianciarullo, 1987).

Em 1986, o planejamento da assistência de enfermagem passa a ser uma imposição legal, por meio da Lei do Exercício Profissional (Lei 7.498), publicada pelo Conselho Federal de Enfermagem (COFEN), Art. 11, inciso I, alínea c, segundo a qual são privativos do enfermeiro "planejamento, organização, coordenação, execução e avaliação dos serviços da assistência de enfermagem" (COFEN, 1986). 
Engajada no movimento pelo melhor direcionamento de suas ações assistenciais, a enfermagem reforça a importância de se planejar a assistência de enfermagem, quando o COFEN aprova a resolução 272/2002, que foi atualizada pela resolução 358, de 15 de outubro de 2009, a qual traz, em seu Art. 1丷, o seguinte: "O Processo de Enfermagem deve ser realizado, de modo deliberado e sistemático, em todos os ambientes, públicos ou privados, em que ocorre o cuidado profissional de Enfermagem" (COFEN, 2009, p.2).

Sua importância na enfermagem se dá por ser um modelo metodológico que evoluiu de identificador de problemas e de suas resolutividades para também identificar e classificar os Diagnósticos de Enfermagem, com foco na especificidade prática e nas intervenções reais de enfermagem que visem a resultados e instiguem produções científicas (Santos, Santos, Lopes, Madeira, \& Rocha, 2014).

De acordo com a resolução 358/2009, Art. 2ํㅡ, o PE é um instrumento metodológico e científico, implementado para favorecer a prestação de um cuidado de qualidade, centrado no indivíduo, na família e na coletividade. Organiza-se em cinco etapas inter-relacionadas, interdependentes e recorrentes, a saber: histórico de enfermagem, Diagnóstico de Enfermagem, planejamento de enfermagem, implementação e avaliação de enfermagem. $A$ resolução ainda determina fundamentar-se em uma teoria que direcione à elaboração de um instrumento para a coleta de dados, ao planejamento das ações e intervenções de enfermagem e ao estabelecimento de Diagnóstico de Enfermagem, e que forneça a base para a avaliação dos resultados de enfermagem alcançados.

No Brasil, Wanda de Aguiar Horta foi responsável pelo emprego do termo e desenvolvimento do PE e o conceituou "como a dinâmica das ações sistematizadas e interrelacionadas visando à assistência ao ser humano, que se caracteriza pelo interrelacionamento e dinamismo de suas fases" (Horta, 2011).

Assim, o PE tem representado o principal modelo metodológico para o desempenho sistemático da prática profissional, com ênfase no raciocínio diagnóstico e no pensamento crítico, ou um instrumento tecnológico de que se lança mão para favorecer o cuidado, organizar as condições necessárias à realização do cuidado e documentar a prática profissional (Garcia \& Nóbrega, 2009), fundamentado em um conjunto de conhecimentos técnicos e baseado em evidências científicas da área da enfermagem. Assim, ele oferece suporte para organizar o processo de trabalho assistencial da enfermagem, tendo como 
referência o cuidado centrado nas necessidades do paciente e devendo articular-se a uma teoria de enfermagem.

Sua aplicação nas instituições de saúde apresenta muitos aspectos positivos, como segurança no planejamento, na execução e na avaliação das condutas de enfermagem; individualização da assistência; visibilidade e autonomia para o enfermeiro; diminuição do tempo de hospitalização e, consequentemente, economia de recursos (Santos et al., 2014; Mola, Dias, Costa, Fernandes, \& Lira, 2019).

Dada à dimensão da problemática em pauta e considerando a necessária adesão dos enfermeiros em busca da qualificação de suas práticas pela adoção de métodos de trabalho embasado cientificamente e capaz de transformar suas realidades, o presente estudo objetivou conhecer o desenvolvimento do processo de implantação do PE em um hospital.

\section{METODOLOGIA}

O artigo é resultado parcial de uma investigação maior, que teve como objetivo geral identificar o desenvolvimento do PE/Sistematização da Assistência de Enfermagem na Rede de Serviços de Saúde de Campo Grande (MS). O estudo ora apresentado foi desenvolvido no Hospital Universitário Maria Aparecida Pedrossian (HUMAP), certificado como hospital de ensino da Universidade Federal de Mato Grosso do Sul (UFMS).

Nesse sentido, a abordagem qualitativa e descritiva de pesquisa delineada pelo método de pesquisa-ação (Thiollent, 2011) foi escolhida como o paradigma metodológico mais adequado para explorar o problema desta pesquisa. Por trabalhar com interação entre pesquisador e participantes, busca identificar um problema em um contexto social/institucional, levantar dados relativos a ele e analisar sua significação, objetivando intervir na prática, no sentido de provocar a transformação. Trata-se, assim, de importante ferramenta metodológica, capaz de aliar teoria e prática por meio de ação que visa transformar determinada realidade. Daí sua importância na área da saúde e enfermagem como instrumento de educação, investigação e mudança (Koerich, Backes, Sousa, Erdmann, \& Alburquerque, 2009).

Tais características determinaram a escolha do método, tendo em conta que a implantação do PE ainda não ocorreu a contento, constituindo motivo de grande inquietação/insatisfação dos profissionais que a defendem. 
O requisito para o desenvolvimento do estudo foi a formação de um grupo de apoio, com representantes das quatro grandes áreas e/ou linhas de cuidado, que compõem a organização do cuidado no hospital, a saber: adulto, materno-infantil, criança e adolescente e pacientes externos.

Esse grupo recebeu o título de Comissão de Sistematização da Assistência de Enfermagem (COMSAE), a qual foi formalmente institucionalizada com a incumbência das formalidades administrativas relacionadas ao funcionamento dos trabalhos os procedimentos de registro detalhado das deliberações ocorridas nos encontros.

O método da pesquisa-ação permitiu um mergulho na realidade de trabalho dos enfermeiros, de forma a promover uma reflexão crítica no modo como aplicavam o PE. Foram percorridas as seguintes etapas da pesquisa-ação: fase exploratória, definição do problema, reconhecimento do lugar da teoria na orientação da prática, hipóteses, seminários, escolha dos sujeitos do estudo (representatividade), coleta de dados, aprendizagem, plano de ação e divulgação externa (Thiollent, 2011). Na execução destas fases, cumpriram-se as características da flexibilidade e a circularidade no desenvolvimento do processo.

Inicialmente, instituiu-se um questionamento com o intuito de provocar um sentimento de incômodo diante da situação de desenvolvimento do PE no hospital, descobrir as expectativas do grupo, realizar o diagnóstico situacional e delimitar os principais problemas e planejamento das ações para resolvê-los.

Mesmo considerando que a pesquisa qualitativa e a pesquisa-ação privilegiam a abordagem empírica (Thiollent, 1986), os encontros sempre foram conduzidos tendo como referência os conhecimentos teóricos em que se fundamenta o PE.

Assim, cada área/linha de cuidado foi chamada para se "olhar no espelho" a partir das seguintes questões norteadoras: Qual a quantidade de PE realizados na unidade, considerando o número de pacientes e de enfermeiros/turnos?; Qual o instrumento aplicado para ao exame físico/histórico?; Quais os critérios utilizados para selecionar o paciente ao qual será aplicado o PE? Como é a forma de registro do PE na unidade?

Essas reflexões foram objeto de apresentações feitas pela COMSAE, promovendo revisões de cunho teórico, para resgate/apropriação de uma teoria de enfermagem que orientasse as 
práticas assistenciais do serviço; reconstrução dos instrumentos/formulários que norteavam as ações/intervenções dos enfermeiros em direção à prática de enfermagem centrada nas necessidades do usuário do serviço, na teoria escolhida e cientificamente evidenciada; revisão da taxonomia de diagnóstico aplicada e reconhecimento do sistema de registro do PE na instituição. Esse foi um movimento de ressignificação da prática e projeção dos resultados que seriam alcançados.

Confirmava-se, assim, o pressuposto de que, na pesquisa-ação, os processos de investigação, educação e ação acontecem simultaneamente. A pesquisa-ação assume, então, caráter emancipatório, no qual os sujeitos da pesquisa passam a ter a possibilidade de se libertarem dos mitos e preconceitos que organizam suas defesas, em direção às mudanças desejadas (Barbier, 2002).

Nesses encontros, foi promovido o gradativo amadurecimento dos participantes da COMSAE o suficiente para que pudessem participar do estudo. A escolha dos sujeitos do estudo foi definida na COMSAE e constituída por 87 enfermeiros assistenciais das quatro linhas de cuidado que aceitaram participar da pesquisa e desenvolviam atividades relacionadas ao PE nas unidades de internação. Foram excluídos enfermeiros que participavam da COMSAE, comissões e cargos na alta direção. Para o cumprimento das fases da pesquisa-ação descritas, manteve-se um cronograma de trabalho rigoroso de reuniões, pautado em muitas discussões com os grupos de enfermeiros, o que exigiu muito tempo e dedicação dos pesquisadores.

Esta pesquisa se realizou aplicando-se um instrumento composto de perguntas objetivas e subjetivas (Polit, 2018). As questões contidas no instrumento contemplaram três dimensões: as características sociodemográficas dos participantes; o conhecimento dos enfermeiros sobre o PE e os aspectos estruturais do serviço que facilitavam ou dificultavam sua aplicação em cada área de atuação desses profissionais. No entanto, as questões subjetivas foram objeto de análise deste estudo. Os participantes foram identificados com a letra $\mathrm{E}$ seguida do número de ordem, para preservar sua identidade.

A coleta de dados transcorreu em 3 dias consecutivos do mês de fevereiro de 2018, nos três períodos de trabalho do enfermeiro simultaneamente, de forma a evitar a divulgação indevida do conteúdo do instrumento. 
Para análise dos conteúdos que emergiram das questões, foi utilizada a técnica da Análise

Temática. As questões foram organizadas em seis categorias, que possibilitaram a interpretação das falas dos sujeitos por meio de análise reflexiva dos conteúdos e das inferências dos pesquisadores, segundo propõe Bardin (2011). A análise temática permitiu identificar as seguintes categorias: Conhecimento do enfermeiro sobre o conceito de Sistematização da Assistência de Enfermagem; Etapas do Processo de Enfermagem; Indicação das fases do Processo de Enfermagem aplicadas diariamente; Habilidade na aplicação da classificação de Diagnóstico de Enfermagem; Fragilidades na aplicação do Processo de Enfermagem.

Este estudo foi aprovado pelo Comitê de Ética em Pesquisa da Universidade Federal de Mato Grosso do Sul, por meio do parecer.133.907/2017.

\section{RESULTADOS}

Participaram do estudo 87 enfermeiros, sendo $77 \%$ do sexo feminino. A média de idade foi de 40,38 anos. Predominaram aqueles que possuíam entre 6 e 10 anos de formação na graduação em enfermagem, totalizando 36 (41\%). Em relação ao tempo de trabalho, 68 (77\%) participantes trabalhavam na instituição entre 1 a 5 anos. A maior parte deles era especialista (89\%) e apenas 2,64 (3\%) eram Mestres. Quanto aos vínculos empregatícios, 75 (85\%) participantes trabalhavam apenas naquela instituição.

\subsection{Conhecimento do enfermeiro sobre o conceito de Sistematização da Assistência de Enfermagem}

A maioria dos participantes $(56,8 \%)$ trouxe um conceito que se aplicava melhor ao PE e não à Sistematização da Assistência de Enfermagem.

"É um processo que envolve as etapas independentes, porém interligadas de maneira organizada e sistematizada, exigindo do enfermeiro conhecimento teórico-científico." (E63)

Poucos sujeitos $(7 \%)$ apresentaram um conceito correto de Sistematização da Assistência de Enfermagem, às vezes aplicando a terminologia implícita na resolução.

"Sistematização da Assistência de Enfermagem é uma forma de organização do serviço para que seja possível a prática do Processo de Enfermagem." (E27) 


\subsection{Etapas do Processo de Enfermagem}

A maioria dos participantes (53\%) relacionou todas as etapas do PE. Porém, um número expressivo deles $(39,1 \%)$ preencheu de modo incompleto as etapas do PE - não empregando a terminologia e/ou não descrevendo a sequência correta. Houve ainda uma parcela de participantes $(2,3 \%)$ que respondeu de forma completamente errada esta questão.

"Coleta de dados; Diagnóstico de Enfermagem; planejamento de enfermagem; implementação; avaliação." (E43)

"Histórico de enfermagem, prescrição de enfermagem." (E01).

“Levantamento de dados, aplicação do processo, Processo de Enfermagem, avaliação, histórico.” (E71)

\subsection{Indicação das fases do Processo de Enfermagem aplicadas diariamente}

As etapas histórico, diagnóstico, prescrição e avaliação foram aplicadas por $53 \%$ dos participantes do estudo.

“(...) Histórico, diagnóstico, prescrição, evolução.” (E15)

"Quatro etapas: diagnóstico, planejamento, implementação e avaliação. O histórico é realizado na admissão." (E51)

\subsection{Habilidade na aplicação da classificação de Diagnóstico de Enfermagem}

Parcela significante dos participantes (41,38\%) declarou possuir habilidade na aplicação da classificação de diagnóstico. Destes, 11 (12,6\%) revelaram ter habilidade na aplicação da North American Nursing Diagnosis Association Internationa (NANDA-I).

"Não tenho dificuldade para utilizar." (E55)

"Me sinto confortável para o uso de diagnóstico segundo o NANDA (...)." (E38)

No entanto, a resposta a esta questão revelou fragilidade do conhecimento do conteúdo relacionado à aplicação da classificação do diagnóstico, pois 21 (24,1\%) participantes não responderam, e outros 19 (21,8\%) declararam não ter habilidade para aplicá-la.

"Dificuldade para encaixar o que foi identificado no paciente dentro das opções propostas." (E17) 


\subsection{Fragilidades na aplicação do Processo de Enfermagem}

Quando foi solicitado ao participante apontar os aspectos que fragilizam a aplicação do PE em sua área de atuação, 36 (31,32\%) deles apontaram deficiência no dimensionamento de pessoal e $17(14,79 \%)$ registraram falta de um sistema de informação que comportasse os registros do PE e, ainda, falta de insumos. Desconhecimento dos profissionais sobre o PE foi apontado por $12(10,44 \%)$ profissionais como fatores que prejudicaram o próprio desempenho.

\section{DISCUSSÕES}

As características sociodemográficas da amostra permitem inferir que o hospital investigado possui atualmente um maior contingente de profissionais de enfermagem do sexo feminino, adultas jovens, com pouco tempo de formação e de trabalho na instituição, especialistas, compondo uma força de trabalho na área da enfermagem com potencial favorável à implementação do PE de forma ampla e qualificada.

Os enfermeiros, em sua maioria, não distinguem claramente e não aplicam corretamente os conceitos de Sistematização da Assistência de Enfermagem e PE, próprios da profissão, o que sugere falta de preparo deles e retrata um enfraquecimento e uma desarticulação da teoria com a prática, gerando conflitos, que prejudicam tanto o entendimento da assistência de enfermagem, quanto a compreensão das teorias de enfermagem. Porém, as observações do emprego de conceitos de forma descuidada e conflituosa constam na literatura da enfermagem. Passos, Santana e Oliveira (2014), ao questionarem enfermeiros se existia diferença entre PE e Sistematização da Assistência de Enfermagem, observaram que $66,7 \%$ dos pesquisados responderam de forma afirmativa, porém a maioria não soube explicar tal distinção. É comum encontrar, na produção científica da enfermagem, iguais conceituações para designar o PE e a Sistematização da Assistência de Enfermagem. Em consequência, muitos pesquisadores acabam por utilizá-las como sinônimos (Oliveira, Almeida, Moreira, \& Torres, 2019). Infelizmente, o resultado da análise dos dados no presente estudo não modifica as constatações de outros autores e implica em sugerir a necessidade de discussão sobre a temática, a fim de se buscar um consenso na linguagem, que promova a qualificação e o aprimoramento da enfermagem, levando em conta as constantes atualizações sobre ela e as mudanças que ocorrem no cenário da assistência (Fuly, Leite, \& Lima, 2008). 
Quanto ao conhecimento dos enfermeiros sobre as etapas do PE, a maioria dos participantes (53\%) relacionou as cinco etapas. Foram consideradas corretas as respostas que referiram que o PE era composto pelas seguintes fases: histórico de enfermagem, Diagnóstico de Enfermagem, planejamento de enfermagem, implementação e avaliação de enfermagem, conforme estabelecido na resolução do COFEN 358/2009 (COFEN, 2009). Na aplicação do PE, os enfermeiros devem seguir rigorosamente suas etapas, para que se possa dizer realmente que o processo está implantado (Santos et al., 2014). A ausência de uma delas torna o PE incompleto, e ele não pode assim ser denominado como tal. Outros autores, quando buscaram identificar o conhecimento dos enfermeiros sobre as fases do PE, encontraram percentuais mais baixos: 31,5\% por Machado et al. (2019) e Félix, Rodrigues e Oliveira (2009) encontraram que 33\% dos enfermeiros conheciam todas as fases. Portanto, o resultado obtido no presente estudo se mostrou animador.

Quando tratam da aplicação das fases do PE, os participantes informaram aplicar predominantemente quatro etapas: histórico, diagnóstico, prescrição e avaliação de enfermagem - por vezes aplicando terminologia diferente da encontrada na resolução, como, por exemplo, "coleta de dados" no lugar de "histórico", "prescrição" no de "planejamento", e "evolução" ao invés de "avaliação", mas que foi considerada correta neste estudo. Oliveira, Coelho, Almeida, Lisboa e Macêdo (2012) também encontraram que o PE existia de forma incompleta, detendo-se no uso de um instrumento impresso e diagnósticos e prescrições diárias. Neves e Shimizu (2010) encontraram, com maior frequência, prescrição e histórico, e, com menor frequência, evolução e Diagnóstico de Enfermagem. Assim, a implantação do PE ocorre de forma ainda bastante fragmentada e com necessidade de se promoverem ações para a conscientização da importância da adoção de um método de trabalho que confira cientificidade, especificidade e autonomia ao trabalho do enfermeiro, tendo em vista o fortalecimento de sua capacidade na tomada de decisão.

Ao avançar na busca de uma linguagem própria da enfermagem, chegou-se ao que, hoje, denominam-se taxonomias. A primeira versão foi publicada em 1990, pela NANDA-I, contendo diagnósticos prontos, divididos em domínios, classes e diagnóstico (Mola et al., 2019). A aplicação do PE à prática assistencial no cotidiano implica o domínio dessa linguagem.

Quando foram solicitados a informar sobre a habilidade na aplicação da classificação de diagnóstico $41,38 \%$ (36) dos participantes declararam possuir habilidade, com destaque 
para a aplicação da NANDA-I. No entanto, outros 45,9\% (40) revelaram não terem habilidade para aplicar a classificação, denotando fragilidade do grupo, em termos do conhecimento do conteúdo relacionado à aplicação da classificação do diagnóstico. $\mathrm{O}$ diagnóstico é inerente à aplicação do PE, pois organiza e sistematiza o julgamento clínico, tendo em vista a tomada de decisão que subsidia a execução do cuidado e possibilita sua avaliação. Esses resultados ainda indicam a necessidade de providências imediatas, em termos de qualificação desses profissionais para prosseguir na implementação do PE, no âmbito do hospital. O uso dos diagnósticos direciona/prioriza a assistência de enfermagem para as necessidades de cada cliente; facilita a escolha de intervenções mais adequadas e urgentes; registra, de forma objetiva, as reações do cliente e permite a subsequente avaliação dos cuidados de enfermagem (Lopes, 2000; Neves \& Shimizu, 2010).

Em relação aos aspectos que fragilizam a aplicação do $\mathrm{PE}, 31,32 \%$ dos enfermeiros apontaram deficiência no dimensionamento de pessoal; outros 14,79 \% registraram a falta de um sistema de informação que comporte todas as etapas dos registros do PE, visto que, no hospital em estudo, parte do PE é registrada manualmente, em formulários impressos, e parte é registrada no sistema de informação. Foi preocupante o fato de o desconhecimento dos profissionais do PE ter sido apontado por 12 (10,44\%) profissionais, como causa de prejuízo à aplicação do PE. Em outros estudos, foi assinalado que o dimensionamento inadequado de pessoal e o não estabelecimento de prioridades na organização do processo de trabalho são dificuldades para a implantação do PE (Machado et al., 2019; Santos et al., 2014). Os resultados do presente estudo se aproximaram dos relatados por estes autores, evidenciando que as dificuldades encontradas não são locais.

A execução do PE conta com amparo de legislação específica, trabalhada na formação do enfermeiro desde a academia. Porém os profissionais ainda encontram dificuldades em cumprir todas as etapas, e algumas foram registradas pelos enfermeiros participantes desse estudo. A não aplicação do método de forma integral leva à fragmentação do cuidado oferecido ao paciente e limita a ação de planejamento de uma assistência resolutiva e que contemple a integralidade do cuidado, além de inviabilizar a realização da experiência de aplicação do método e da crença na aplicação do mesmo.

\section{CONCLUSÕES}

O estudo revelou que o conhecimento dos enfermeiros em relação aos conceitos próprios da profissão é frágil, apresentando por vezes equívoco na aplicação dos conceitos de 
Processo de Enfermagem e Sistematização da Assistência de Enfermagem. Essa dificuldade com a aplicação dos conceitos é reconhecida na literatura de enfermagem.

A maioria dos enfermeiros indicou as cinco fases propostas para a aplicação do Processo de Enfermagem, porém foi registrado o emprego de denominações diferentes das propostas na legislação de enfermagem. Quando se tratou da aplicação dessas etapas, a informação predominante foi de que eram aplicadas quatro delas.

Ao apresentarem respostas sobre possuir habilidade para aplicação da classificação de diagnóstico, a maioria revelou não tê-la, denotando fragilidade dos participantes em termos do conhecimento desse conteúdo. Foram encontradas deficiência no dimensionamento de pessoal e sobrecarga de trabalho como fatores que prejudicam o desempenho dos enfermeiros. Também faltava um sistema de informação que comportasse todas as etapas dos registros do Processo de Enfermagem. Estas colocações evidenciam a necessidade de se (re)conhecer a estrutura institucional na qual o Processo de Enfermagem está implantado e garantir algumas condições, como, por exemplo, número suficiente de enfermeiros para essa prática.

Considerando o estágio de desenvolvimento da implantação do Processo de Enfermagem no hospital em estudo, o grupo de enfermeiros precisa buscar caminhos que possam lhes ajudar a sensibilizar as chefias de enfermagem e a diretoria do hospital para que acreditem que o Processo de Enfermagem constitui a estratégia mais adequada para promover a melhora da qualidade da assistência de enfermagem ao paciente. Deve também promover a valorização dos profissionais de enfermagem.

Este estudo possibilitou evidenciar a importância da utilização da pesquisa-ação como estratégia que induz o reconhecimento dos problemas relacionados à aplicação do Processo de Enfermagem na prática assistencial dos enfermeiros e contribuiu para o desenvolvimento do conhecimento e habilidades dos participantes da Comissão de Sistematização da Assistência de Enfermagem.

Acreditamos na potencialidade das estratégias que fortalecem as práticas de integração entre ensino e serviço e o uso da metodologia da pesquisa-ação como estratégia de aproximação entre os pesquisadores e os enfermeiros do hospital. Esperamos contribuir para a implementação do Processo de Enfermagem no serviço envolvido no estudo. 
Pesquisas como esta são importantes por demonstrarem as aproximações possíveis da prática do enfermeiro, bem como a necessidade de fortalecimento de seu papel na promoção da qualidade do cuidado. Deve haver mais envolvimento institucional, oferecendo apoio para que a configuração dos processos de trabalho da enfermagem seja favorável às melhores práticas.

\section{REFERÊNCIAS}

Andrade, J. S., \& Vieira, M. J. (2005). Prática assistencial de enfermagem: problemas, perspectivas e necessidade de sistematização. Revista Brasileira de Enfermagem, 58(3), 261-265.

Barbier, R. (2002). A pesquisa-ação. Brasília, DF: Plano.

Bardin, L. (2011). Análise de conteúdo. São Paulo: Edições 70.

Barret, E. A. M. (2002). What is nursing science? Nursing Science Quarterly, 15(1), 51-60.

Carper, B. A. (1978). Fundamental patterns of knowing in nursing. Advance in Nursing Sicence, 1(1), 13-24.

Cianciarullo, T. I. (1987). Teoria das necessidades humanas básicas - um marco indelével na enfermagem brasileira. Revista da Escola de Enfermagem da USP, 21, 100-107.

Conselho Federal de Enfermagem (COFEN). (2009). Resolução COFEN-358/2009. Brasília, DF: COFEN. Disponível em: http://www.cofen.gov.br/resoluo-cofen-3582009_4384.html

Conselho Federal de Enfermagem (COFEN). (1986). Lei n. 7.498, de 25 de junho de 1986. Brasília, DF: Diário Oficial da União. Disponível em: http://www.cofen.gov.br/lei-n-749886-de-25-de-junho-de-1986_4161.html

Félix, N. N., Rodrigues, C. D. S., \& Oliveira, V. D. C. (2009). Desafios encontrados na realização da Sistematização da Assistência de Enfermagem (SAE) em unidade de pronto atendimento. Arquivos de Ciências da Saúde, 16(4), 155-160.

Friedlander, M. R. (1981). O processo de enfermagem ontem, hoje e amanhã. Revista da Escola de Enfermagem da USP, 75(2), 129-134.

Fuly, P. S. C., Leite, J. L., \& Lima, S. B. S. (2008). Correntes de pensamento nacionais sobre sistematização da assistência de enfermagem. Revista Brasileira de Enfermagem, 61(6), 883-887.

Garcia, T. R., \& Nóbrega, M. M. L. (2009). Processo de Enfermagem: da teoria à prática. Escola Anna Nery Revista de Enfermagem, 13(1), 188-193.

Horta, W. A. (2011). Processo de Enfermagem. 14 ed. São Paulo: EPU.

Koerich, M. S., Backes, D. S., Sousa, F. G. M., Erdmann, A. L., \& Alburquerque, G. L. (2009). Pesquisa-ação: ferramenta metodológica para a pesquisa qualitativa. Revista Eletrônica de Enfermagem, 11(3), 717-723.

Lopes, M. H. B. de M. (2000). Experiência de implantação do Processo de Enfermagem utilizando os Diagnósticos de Enfermagem (taxonomia da Nanda), resultados esperados, intervenções e problemas colaborativos. Revista Latino-Americana de Enfermagem, 8(3), 115-118.

Machado, J. P. C., Silva, D. M., Souza, E., Pedron, C. D., Gallash, C. H., \& Thiengo, P. C. da S. (2019). Percepção de enfermeiros de unidade de internação clínica sobre a Sistematização da Assistência de Enfermagem. Revista Nursing, 22(257), 3220-3225.

Marques, S. M., Brito, K. C. G.; Fernandes, C. M., \& Vieira, A. G. (2008). Sistematização da assistência de enfermagem na UTI: perspectivas dos enfermeiros da cidade de Governador Valadares. Reme - Revista 
Mineira de Enfermagem, 12(4), 469-476.

McEwen, M., \& Wills, E. M. (2016). Bases teóricas de enfermagem. 4 ed.. Porto Alegre, RS: Artmed.

Mola, R., Dias, M. L., Costa, J. F., Fernandes, F. E. C. V., \& Lira, G. G. (2019). Conhecimento dos profissionais de enfermagem sobre a sistematização da assistência de enfermagem. Revista Cuidado é Fundamental, 11(4), 887-893.

Neves, R. de S., \& Shimizu, H. E. (2010). Análise da implementação da Sistematização da Assistência de Análise da implementação da Sistematização da Assistência de Enfermagem em uma unidade de reabilitação. Revista Brasileira de Enfermagem, 63(2), 222-229.

Oliveira, M. R., Almeida, P. C., Moreira, T. M. M., \& Torres, R. A. M. (2019). Nursing care systematization: perceptions and knowledge of the Brazilian nursing. Revista Brasileira de Enfermagem, 72(6), 1547-1553.

Oliveira, A. P. C., Coelho, M. E. A. A., Almeida, V. C. F., Lisboa, K. W. S. C., \& Macêdo, A. L. S. (2012). Sistematização da assistência de enfermagem: implementação em uma unidade de terapia intensiva. Revista Rene, 13(3), 601-612.

Passos, K. dos S., Santana, M. O., \& Oliveira, C. G. S. de. (2014). Percepção dos enfermeiros na implantação da sistematização da assistência de enfermagem em um hospital filantrópico. Interfaces Científicas - Saúde e Ambiente, 2(3), 53-62.

Polit, D. F. (2018). Fundamentos de pesquisa em enfermagem avaliação de evidências para a prática da enfermagem (9 ed.). Porto Alegre: ArtMed.

Santos, W. N. dos, Santos, A. M., Lopes, T. R. P., Madeira, M. Z., \& Rocha, F. C. (2014). Sistematização da Assistência de Enfermagem: o contexto histórico, o processo e obstáculos da implantação. Journal of Management and Primary Health Care, 5(2), 153-158.

Thiollent, M. (2011). Pesquisa-ação nas organizações. São Paulo: Cortez.

Thiollent, M. (1986). Metodologia da pesquisa-Ação. São Paulo: Cortez. 